\title{
Das Korpus des Projekts "Mischphänomene zwischen Dialekt und Standardsprache in der Deutschschweizer Diglossie". Über die Schwierigkeit, selten vorkommende Phänomene zu dokumentieren
}

\author{
Marina Petkova (Fribourg)
}

\begin{abstract}
An ongoing research project at the University of Fribourg/Freiburg (Switzerland) investigates relatively infrequent forms of usage of the dialect and the standard variety of a language. The project focuses on verbal realisations in which both varieties exist side by side but which do not constitute cases of code-switching in the traditional sense; that is, the code changes do not serve a local pragmatic function and/or their salience has diminished as a result of their high frequency of occurrence. Furthermore, the mixing occurs at conspicuous syntactic positions, and the transferred linguistic elements do not rank among the typical resources for codeswitching. Moreover, the resulting realisations - if decontextualised - may tend to be judged as impermissible by autochthonous speakers. Such phenomena are unexpected in Swiss German diglossia and contradict not only lay conceptions but also an assumption underlying many scientific investigations which states that speakers of Swiss German keep the two varieties strictly distinct in their usage.
\end{abstract}

This contribution will shed light on several aspects of corpus design, with a special focus on the difficulties involved in compiling a corpus for the documentation of rare phenomena.

\section{Ausgangslage und Fragestellungen}

Die sprachliche Situation in der deutschsprachigen Schweiz wird allgemein mit dem Begriff der Diglossie beschrieben. Nach der von Ferguson (1959) geprägten Definition ist dies der Gebrauch zweier genetisch verwandten Varietäten, die von der gesamten Sprachgemeinschaft beherrscht und in verschiedenen Domänen mit unterschiedlicher Funktion verwendet werden. ${ }^{1}$

Die Kontexte, in denen beide Varietäten jeweils zum Zuge kommen, haben sich im Verlauf der Zeit infolge gesellschaftlicher und technischer Veränderungen gewandelt. So wurde die von Ferguson postulierte Verteilung der Varietäten nach bestimmten Domänen (Kirche, Politik, Familie etc.) später zu einer als "mediale Diglossie" (Kolde 1981) bezeichneten Verteilung auf die Medien der Schriftlichkeit (Standardsprache) und der Mündlichkeit (Dialekt), während in neuerer Zeit von einer Verteilung auf der Achse zwischen Nähe und Distanz bzw. Formalität und Informalität ausgegangen wird (u. a. Ammon et al. 2004). Der Einzug der elektronischen Kommunikation (SMS, E-Mail, Chat etc.) in den Alltag und die erhöhte Ak-

\footnotetext{
${ }^{1}$ Auf den umstrittenen Aspekt des höheren und niedrigeren Prestige der L- und H-Varietät wird an dieser Stelle nicht näher eingegangen.
} 
zeptanz gegenüber informelleren Registern in den Printmedien ${ }^{2}$ hat es mit sich gebracht, dass der Dialekt vermehrt auch geschrieben wird. Gleichzeitig mehren sich aber auch die Situationen, in denen DeutschschweizerInnen in Kontakt mit allochthonen Nicht-Dialektsprechern kommen und somit die Standardsprache auch als gesprochene Varietät auftritt.

Nie in Frage gestellt wurde die strukturell getrennte Verwendung beider Varietäten und die Trennung nach Kontexten überhaupt. So gehören nach Ulrich Ammon "das penible strukturelle Auseinanderhalten von Dialekt und Standardvarietät" und "die ziemlich konsequente funktionale Trennung von Dialekt und Standardvarietät nach ihrem Gebrauch in den Domänen" (Ammon 1995: 286) zu den wichtigsten Besonderheiten der Sprachverwendung in der deutschsprachigen Schweiz. Noch im Artikel zur "Domänenverteilung zwischen Dialekt und deutscher Standardsprache" des HSK-Bandes Dialektologie wird die in der Schweiz bestehende "scharfe Trennung zwischen dem Schweizerhochdeutschen (Standard) und den schweizerdeutschen Dialekten" als einer der wichtigsten Unterschiede zwischen der schweizerischen und der deutschen/österreichischen Situation angegeben (Schuppenhauer/Werlen 1983: 1442). Und auch das Variantenwörterbuch des Deutschen hält fest: "Dialekt und Standardsprache sind strukturell und psychologisch klar getrennt" und "Dialekt und Standardsprache haben verschiedene, deutlich getrennte Funktionen" (Ammon et al. 2004: XLII). Wechsel zur Standardsprache innerhalb einer dialektal-mündlichen Äusserung dienen in der autochthonen Sprachgemeinschaft als metaphorische "Kontextualisierungshinweise", d. h. als Mittel, um Informationsmehrwert zu signalisieren und "um Schemata aus dem Hintergrundwissen verfügbar zu machen" (Auer 1986: 24). ${ }^{3}$

Die scharfe Trennlinie zwischen den Varietäten ist auch wesentlicher Bestandteil der laienlinguistischen Wahrnehmung der sprachlichen Situation in der Deutschschweiz. Immer wieder wird das Verhältnis zwischen den Varietäten als eine Art Zweisprachigkeit beschrieben, häufig ist aus Laienseite die Forderung nach der Verwendung eines "reinen" Dialektes zu hören, d. h. vor allem eines Dialektes ohne jegliche standardsprachliche Einmischungen (Näheres hierzu in Haas 2004: 93f.) und auch die Standardsprache wird einerseits als Bestandteil von Stereotypen über des Schweizers Abneigung und Unfähigkeit, "das Hochdeutsche" zu sprechen, thematisiert, andererseits als eine Schlüsselkompetenz in der Bildung, deren Erwerb, insbesondere im schulischen Kontext, durch die ständige Präsenz des Dialekts gefährdet sei.

Dass diese ausgesprochen dichotome Sicht in verschiedener Hinsicht an der Realität vorbeigeht, ist bekannt. So teilen beide Varietäten ein Grossteil des Lexikons. Entlehnungen von sprachlichem Material aus der Standardsprache in einer morphologisch und phonetisch angepassten Form sind auch ein verbreitet eingesetztes Mittel, um die Einsatzfähigkeit des Dialekts in allen gesellschaftlichen Kontexten und Themenbereichen zu gewährleisten. Dass dabei sowohl Fachsprachen als auch einige (v. a. gehobene) Sprachregister die Ausprägung eines "suboptimalen Dialekts" (Christen 2000) haben, stört die Sprachpfleger, ermöglicht aber das Entstehen eines "ausgebauten" (Kloss 1976) und somit auch überlebensfähigen Dialekts. Doch nicht diese Form von 'Durchmischung' beider Varietäten ist das Thema der hier vorgestellten Untersuchung. Viel mehr werden sprachliche Realisierungen in den Blick genommen, bei denen beide Varietäten in ihrer erkennbar eigenen Form nebeneinander stehen, bei denen es sich aber nicht um Fälle von code-switching im üblichen Sinne handelt (zum theoretischen Hintergrund hierzu vgl. Auer 1999). Den Code-Wechseln kommt also nicht im einzelnen Fall eine lokale pragmatische Funktion zu und/oder sie haben durch ihre Häufigkeit an Salienz eingebüsst. Auch sind die syntaktischen Stellen, an denen sie auftreten, auffällig und es werden sprachliche Elemente transferiert, die nicht zu den typischen Ressourcen für code-

\footnotetext{
2 Vor allem in Anzeigen und nicht redaktionellen Beiträgen.

${ }^{3}$ Näheres hierzu in Christen et al. (in Vorb.).
} 
switching zählen. Es entstehen somit Realisierungen, die - ihres Kontextes enthoben - von autochthonen Sprechern als unzulässig empfunden würden. Code-switching-Insertionen aus der Standardsprache haben typischerweise die Form von Phraseologismen, Zitaten, Termini, Diskursmarkern und Interjektionen, sie dienen u. a. dazu, Aspekte des Gesagten zu unterstreichen, die Wahl eines Gesprächspartners anzuzeigen etc.; auf der strukturellen Ebene werden vor allem Inhalts- und kaum Funktionswörter transferiert (vgl. Gumperz 1982, Häusermann/Buhofer 1982, Werlen 1988, Bürkli 1999, Christen 2000, Dirim/Auer 2004). Verschiedene Äusserungen im Korpus entziehen sich jedoch einer solchen Beschreibung. Einige Beispiele solcher Realisierungen sind Äusserungen wie: "Ja, da gseht ma schon die Anspannung", "Patrouille isch im Momänt noch besetzt, falls sie frei isch, schicke ich sie zu Ihne(n)", "Wär hat nəch geschlagen?", "Es geht um Aktuelles, wo mir befragen", "De hät khä unglücklichə Iidruck gmacht, also de kann leben mit dem Unentschieden". Die Wechsel der Varietät können Mischphrasen wie diese konstituieren, sie können sich auch durch den Verlauf eines ganzen Gesprächs hindurch ziehen oder aber kleinere Grössen in Form von Hybridwörtern u. ä. betreffen.

Wie sind solche Phänomene in der Deutschschweizer Sprachlandschaft einzuordnen? In welchen Kontexten kommen sie vor und was zeichnet ihre strukturelle Ausprägung aus? Handelt es sich dabei um grundlegende Veränderungen in der Diglossiesituation oder um das Entstehen neuer gesellschaftlicher Kommunikationsmuster? Diese und ähnliche Fragen stehen im Zentrum der hier vorgestellten Untersuchung. ${ }^{4}$ In diesem Beitrag sollen einige Aspekte der Korpuserstellung diskutiert werden, vor allem im Hinblick auf die Schwierigkeit, die damit verbunden ist, ein Korpus zusammenzustellen, in dem seltene Phänomene belegt sind.

\section{Das Korpus}

Um überprüfen zu können, welchen Stellenwert und welche Ausprägung die oben beschriebenen Phänomene im Deutschschweizer Alltag haben, bedarf es als erstes der Zusammenstellung eines Korpus, in dem das Vorkommen solcher Erscheinungen in verschiedenen Kontexten belegt ist. Dabei erweist sich das bekannte "Beobachterparadoxon"5 als zentrale Schwierigkeit. Authentische, von der Aufnahmesituation nicht beeinflusste Daten, sind eine unabdingbare Voraussetzung, insbesondere auch um bisher wenig belegte sprachliche Erscheinungen nachzuweisen. Die verschiedenen Teilkorpora, aus denen unser bisher aufge-bautes Korpus besteht, versuchen dieser Problematik Rechnung zu tragen. Wir sind von einigen grundsätzlichen Möglichkeiten der Datensammlung ausgegangen. Einerseits bieten sich Gespräche an, die nicht für Untersuchungszwecke, sondern zum Zweck einer eventuellen späteren (v. a. internen) Kontrolle in einer Institution oder in einem Unternehmen aufgenom-men worden sind. Andererseits wäre es möglich, Daten eigens für das Forschungsprojekt aufzunehmen, wobei hier bereits mit Annahmen über die Voraussetzungen, unter denen das untersuchte Phänomen vorkommt, gearbeitet werden muss. Und nicht zuletzt dokumentieren Gespräche und Diskussionen in den Medien (Radio und Fernsehen) ein breites Spektrum an Sprachverwendungsformen, die auch für die Sprachgemeinschaft typisch sind.

\subsection{Korpus "Polizeinotruf"}

Zu Beginn konnten wir auf die Daten eines abgeschlossenen Forschungsprojekts zurückgreifen, das an der Universität Freiburg (Schweiz) von 2005 bis 2008 durchgeführt wurde (hierzu

\footnotetext{
${ }^{4}$ Die Untersuchung wird vom Schweizerischen Nationalfonds unterstützt und läuft seit dem Sommer 2008 an der Universität Freiburg (Schweiz) unter dem Arbeitstitel "Mischphänomene zwischen Dialekt und Standardsprache in der Deutschschweizer Diglossie" als Teil des Forschungsprojekts "Die Hochdeutschs in der Schweiz" unter der Leitung von Prof. Helen Christen.

5 "To observe the way people use language when they are not being observed" (Labov 1972: 61).
} 
vgl. Christen et al. (in Vorb.)). Das Korpus besteht aus Aufnahmen von Telefongesprächen, die auf die Polizeinotrufnummer 117 eingegangen sind. In einigen Gesprächen mit allochthonen Anrufenden, also mit Anrufenden, die keinen Schweizer Dialekt als L1 sprechen, haben wir das Auftreten von Mischformen zwischen Dialekt und Standardsprache in der Rede der autochthonen Polizisten beobachtet. Dass es sich dabei nicht um eine Form von foreigner talk handelt, machen diejenigen Beispiele deutlich, die aus Gesprächen mit deutschen Anrufenden jenseits der Landesgrenze stammen. Bei solchen Anrufenden handelt es sich meist um Polizisten - also Kollegen - die häufig selbst Elemente eines (deutschen) alemannischen Dialektes in ihrer Rede aufweisen und zudem durch den häufigen Kontakt mit ihren schweizerischen Kollegen oder Nachbarn mit Sicherheit genügend Kompetenz sowohl in Bezug auf den Schweizer Dialekt als auch auf die Standardsprache mitbringen, um keiner Verständigungshilfe zu bedürfen. Vielmehr haben wir es bei allen Gesprächen dieser Art im Polizeinotrufkorpus mit Gesprächsteilnehmerkonstellationen zu tun, in denen Aspekte der Nähe und Distanz in irgendeiner Form in Widerspruch zueinander stehen oder aber in denen die Wahl einer der beiden Varietäten durch andere Umstände erschwert ist.

Hier als Beispiel ein Gespräch 6 aus dem Korpus:

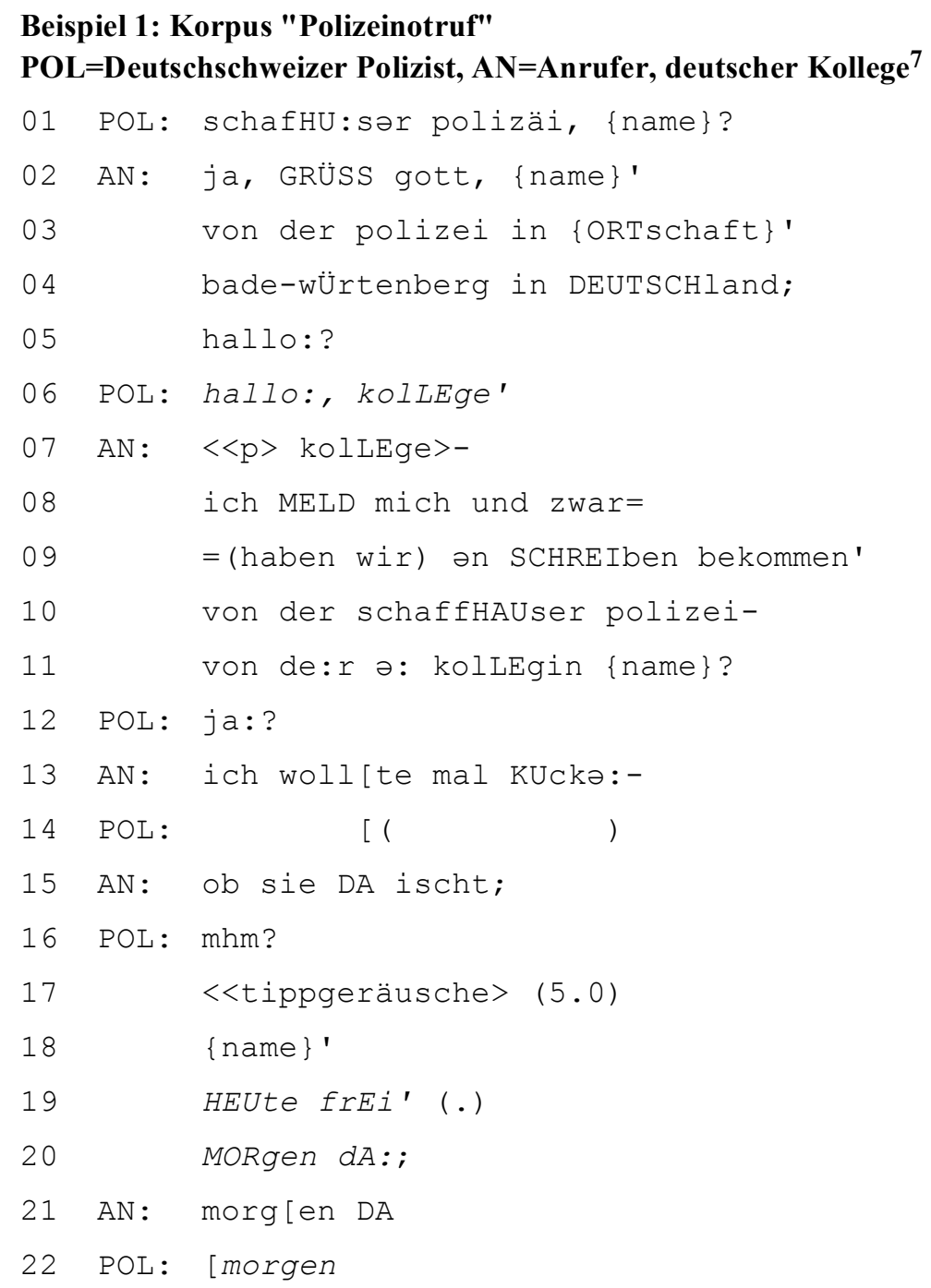

\footnotetext{
6 Als Besonderheit dieses Gesprächs erweist sich die Tatsache, dass der Schweizer Polizist nicht nur Elemente aus der Standardsprache und Elemente aus dem Schweizer Dialekt in seiner Rede mischt, sondern dass er auch einzelne Elemente aus dem Dialekt des Anrufers verwendet.

${ }^{7}$ Standardsprachliche Elemente in der Rede des Schweizer Polizisten wurden kursiv gesetzt.
} 


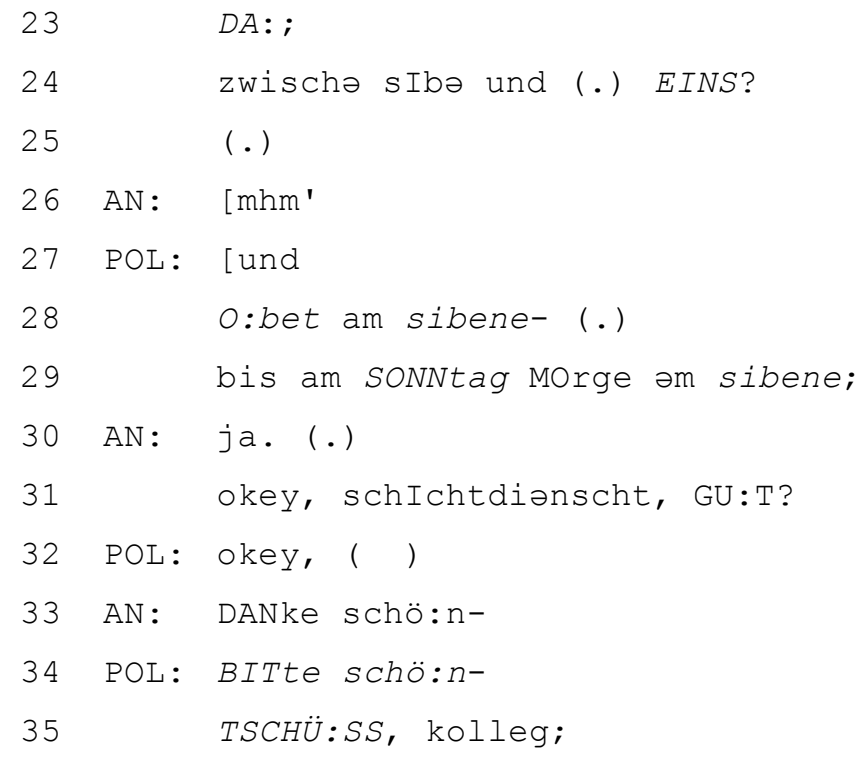

Das Korpus besteht aus der Gruppe der 468 Gespräche mit allochthonen Anrufenden, die aus ursprünglich insgesamt über 6300 Gesprächsdateien aufgrund der Sprechekonstellation ausgewählt wurden. Die Daten des Polizeinotrufs haben sich als für eine linguistische Analyse sehr gut geeignet erwiesen. Die aktivierten Aufnahmegeräte gehören zum beruflichen Alltag der PolizistInnen und werden von ihnen kaum noch bewusst wahrgenommen, sodass sich die Aufnahmen durch hohe Authentizität auszeichnen. Durch die konstante Teilnehmerkonstellation (Polizist - Anruferin) und die ähnlichen Voraussetzungen (Themenbezogenheit, Formalitätsgrad, Gesprächstyp etc.) gewährleisten die Daten aber auch eine sehr gute Vergleichbarkeit untereinander.

\subsection{Korpus "GFS-Kontaktgespräche"}

Die guten Erfahrungen mit dem Datenmaterial der Polizeinotrufgespräche haben gezeigt, dass Aufnahmen von Telefongesprächen, die in einem anderen Kontext, aber unter ähnlichen Voraussetzungen entstanden sind, eine gute Korpusergänzung darstellen würden, so z. B. Aufnahmen von Anrufen, die auf Call-Center- oder Kundendiensttelefonnummern eingegangen sind. Anfragen bei Unternehmen wie Sunrise oder Swisscom wurden leider abgewiesen. Das Befragungsinstitut GFS mit Sitz in Zürich und Bern hat sich jedoch bereit erklärt, dem Projekt Gesprächsaufnahmen zur Verfügung zu stellen. Dabei handelt es sich um 20459 Dateien, Aufnahmen sog. Kontaktgespräche, die der Durchführung von Umfragen zu bestimmten Themen vorausgehen. Der Ausrichtung des Instituts entspricht, möglichst viele, verschiedene Alters- und Bevölkerungsgruppen zu befragen, um repräsentative Resultate zu erreichen, wobei sich die Anrufe an zufällig gewählte, in der Schweiz wohnhafte Personen richten. Die grosse Anzahl Dateien erhöht die Wahrscheinlichkeit, dass Phänomene, die Teil des Deutschschweizer Sprachlebens sind, auch innerhalb eines solchen Korpus Niederschlag gefunden haben könnten. Andererseits beinhaltet diese grosse Datenmenge eine beträchtliche Anzahl Kurzgespräche, in denen die GFS-Angestellten in vorformulierten Sätzen den Zweck ihres Anrufs gleichsam vortragen und umgehend abgewiesen werden. Auch wurden uns aus Datenschutzgründen die eigentlichen Befragungsgespräche, die einer solchen Kontaktaufnahme folgen, nicht zur Verfügung gestellt. Häufig kommt aber die Interaktion erst nach der ersten Kontaktaufnahme in Gang und somit sind mögliche Belege für bestimmte sprachliche Erscheinungen erst in den uns nicht zugänglichen Gesprächsfortsetzungen zu erwarten.

Als erstes Fazit kann festgehalten werden, dass das Korpus "GFS-Kontaktgespräche" durchaus Beispiele für die gesuchten sprachlichen Mischphänomene enthält. Diese sind aber, gemessen an der Gesamtzahl der Dateien, nicht so zahlreich (zum Problem der quantitativen 
Auswertung s. u.). Die Streuung der Belege im Korpus wird es jedoch zusätzlich ermöglichen, Annahmen über die notwendigen Voraussetzungen zu formulieren, unter welchen das Auftreten der untersuchten Phänomene zu erwarten ist.

Auch hier als Beispiel ein Ausschnitt aus einem Kontaktgespräch:

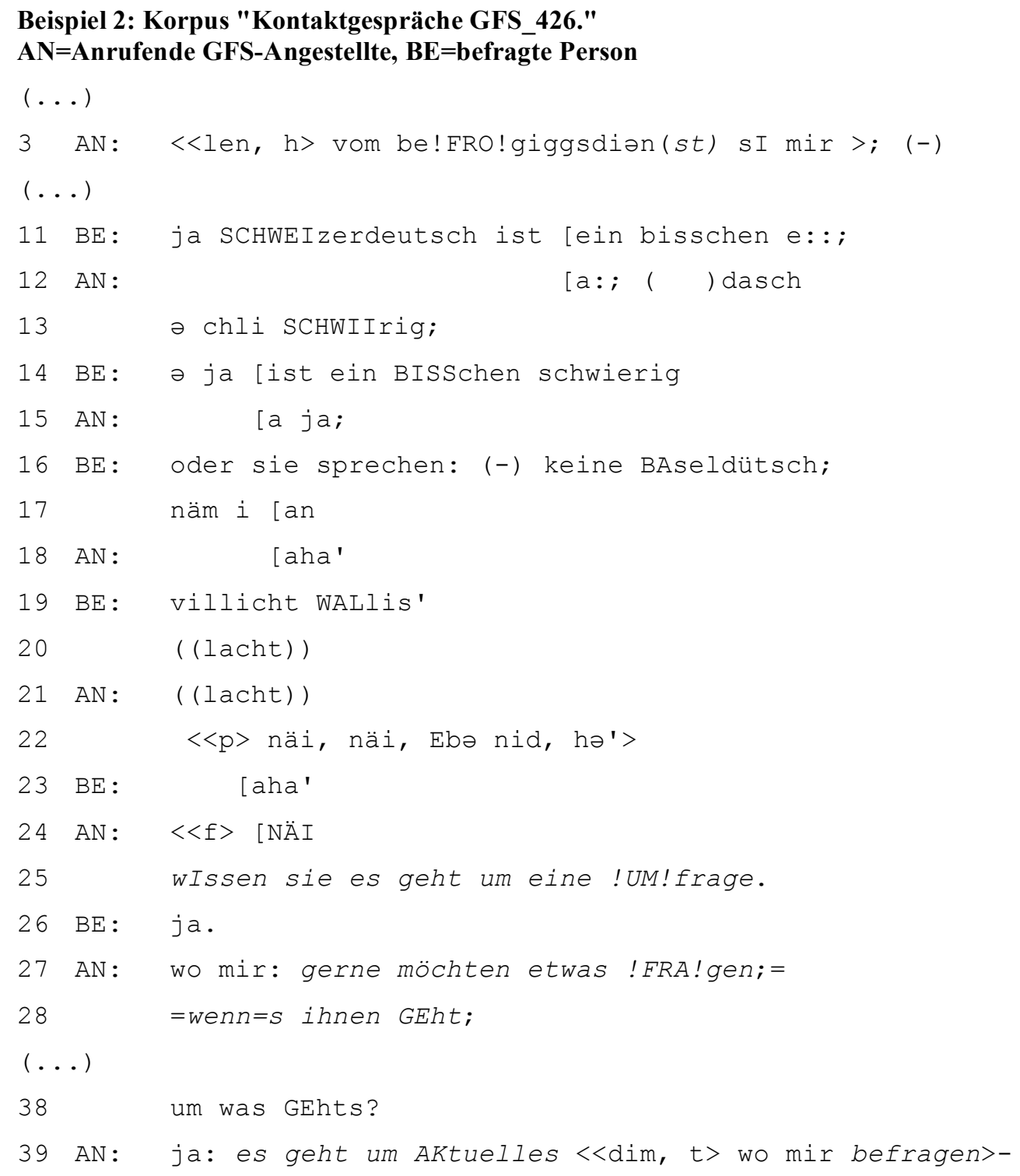

\subsection{Korpus "Studiogespräche Euro 08"}

Ein weiteres Teilkorpus der Untersuchung besteht aus den Gesprächen, die der Fernsehmoderator Rainer Maria Salzgeber während der 'Euro 08' mit Fussballexperten im Studio geführt hat. Die Fernsehübertragungen der Fussball-Europameisterschaft wurden von Studiosendungen begleitet, in denen die Spiele ausführlich kommentiert und mit Hintergrundinformationen angereichert wurden. Dabei besteht an dreizehn der neunzehn Spieltagen das Expertenduo im Studio aus einem allochthonen (jeweils zwei Deutsche und ein Franzose) und einem autochthonen Gast. Die primäre Adressierung an das Deutschschweizer Fernsehpublikum, aber auch die Teilinteraktion mit dem autochthonen Experten bringen es mit sich, dass der Dialekt in allen Studiosendungen als Hauptvarietät fungiert. Dies erfordert auch, dass die geladenen allochthonen Gäste den Dialekt zumindest verstehen sollten und in der Tat kommt es in den Gesprächen selten zu Verständigungsschwierigkeiten in diesem Zusammenhang. Auch wird der Dialekt in direkt an die allochthonen Gäste adressierten Äusserungen seitens des Modera- 
tors häufig beibehalten. Häufig mischt er jedoch beide Varietäten auf recht auffällige Art, wobei dies von der Verwendung einzelner Mischphrasen bis hin zu frequenten Wechseln zwischen beiden Varietäten über längere Gesprächsstrecken reicht.

Als Beispiel zwei Äusserungen Salzgebers aus dem Korpus:

Beispiel 3: Korpus "Euro 08 Studiogespräche"; S=Rainer Maria Salzgeber

\section{Sendung vom 17.06.2008, Min 3.59.00}

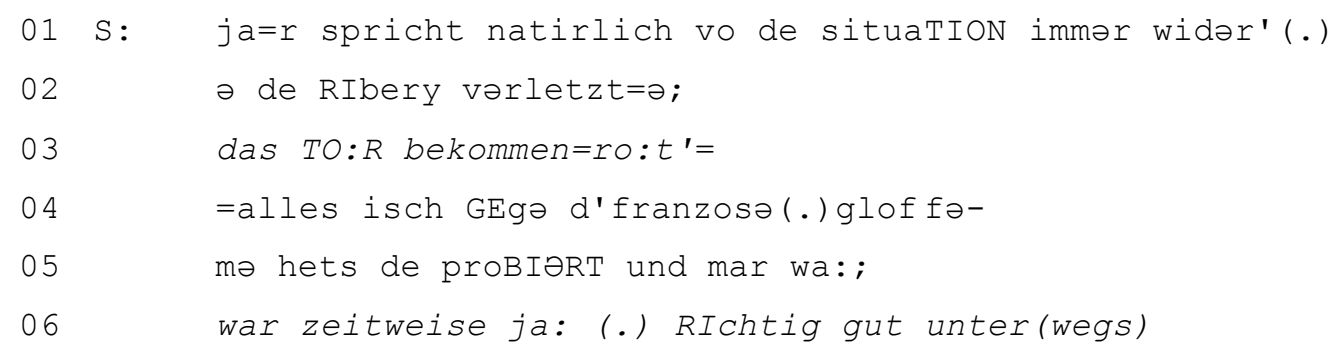

Sendung vom 11.06.2008, Min 03.00.00

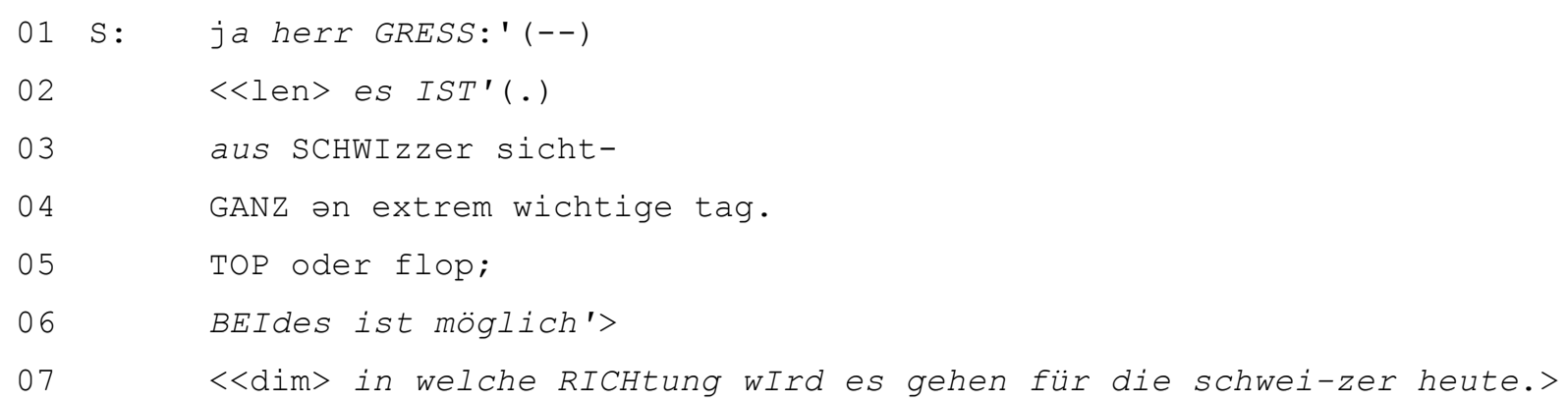

$\mathrm{Zu}$ den Charakteristika des Teilkorpus "Studiogespräche Euro 08" gehört die Tatsache, dass wir es hier mit einer Sprachverwendung zu tun haben, die im öffentlichen Raum stattfindet. Die recht ausführlichen Studiosendungen wurden vom ersten öffentlichen Fernsehsender vor dem jeweiligen Spiel, in der Spielpause und nach dem Spiel ausgestrahlt und erfreuten sich einer konstant hohen Zuschauerquote. In Bezug auf die Untersuchung der sprachlichen Mischphänomene kann festgehalten werden, dass das Korpus eine grosse Anzahl Belege enthält, die vom gleichen Sprecher realisiert werden, was gute Vergleichbarkeit in Bezug auf Funktionen und Struktur erlauben wird. Im Vergleich zu den anderen Teilkorpora, in denen gemessen an die Gesamtzahl Daten weniger Belege enthalten sind, die aus verschiedenen Kontexten und Themenbereichen und von verschiedenen Sprechern stammen, haben wir es somit mit einem Korpus ganz anderer Ausprägung zu tun.

Der bisherige Verlauf der Untersuchung und auch eigene Feld-Beobachtungen legen die Vermutung nahe, dass die Mischphänomene am häufigsten in der face-to-face Interaktion zwischen Autochthonen und Allochthonen in "Redekonstellationen" (Steger et al. 1974) zu erwarten sind, die eine unmittelbare, unvorbereitete, häufig vielleicht auch im privaten Rahmen stattfindende Kommunikation zwischen SprecherInnen gleichen Ranges beinhalten. Ein Desiderat bleibt in diesem Sinne ein Korpusbestandteil, der das Auftreten der untersuchten Phänomene in der unmittelbaren, privaten Interaktion dokumentieren würde. Es liegt in der Natur der Sache jedoch, dass authentische Datenaufnahmen, die auch dem Persönlichkeitsschutz Rechnung tragen, gerade in diesen Konstellationen schwer erhältlich sind. Bisher ist es uns nicht gelungen, entsprechende Aufnahmen für das Forschungsprojekt in die Wege zu leiten, doch vielleicht gelingt es uns, das Korpus in diesem Sinne zu vervollständigen. 


\subsection{Belegsammlung "Mixed code in der Schriftlichkeit"}

Eine Belegsammlung, die das Auftreten von Mischungen beider Varietäten in der Schriftlichkeit abbildet, soll als Vergleichsbasis dienen und das Aufkommen dieser Phänomene sozusagen unter umgekehrten Vorzeichen in den Blick nehmen. So finden sich Beispiele für die Verwendung eines mixed code zwischen Dialekt und Standardsprache z. B. in Restaurantmenus, in Werken der fiktionalen Literatur, in Zeitungen und natürlich an verschiedener Stelle im Internet, in besonders ausgeprägter Form z. B. in der Gestaltung der Homepage der Musikgruppe "Friedli \& Fränz Kilbimusig". Hier ein Beispiel:

Beispiel 4: http://www.holzbein.ch. Kopiert am 4.05.09.8

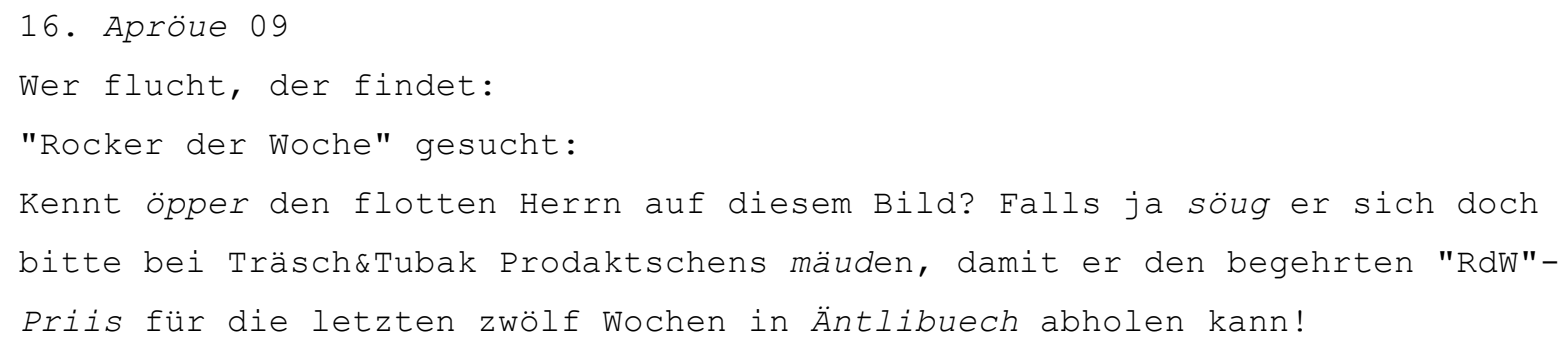

Wie sind solche Phänomene auch im Vergleich zur gesprochenen Sprache zu deuten, gibt es Ähnlichkeiten in der Funktion, können aufgrund ihrer Analyse Aussagen über Entwicklungen in der sprachlichen Situation in der Deutschschweiz formuliert werden? Mit solchen Fragen wird sich die laufende Untersuchung noch beschäftigen.

\section{Problematik der Korpuskonstitution}

Bevor einige wichtige Aspekte der Korpuserstellung und ihrer Problematik angesprochen werden, hier nochmals das gesamte Korpus im Überblick:

\begin{tabular}{|c|c|}
\hline $\begin{array}{l}\text { Korpus „Polizeinotruf“6 } \\
6381 \text { Gesprächsaufnahmen aus } 15 \\
\text { Polizeikommandos }\end{array}$ & $\begin{array}{l}\text { Korpus „,GFS-Kontaktgespräche“6 } \\
20459 \text { Gesprächsdateien } \\
\text { vorsortiert nach Grösse }\end{array}$ \\
\hline $\begin{array}{l}468 \text { Gespräche mit al- } \\
\text { lochthonen Anrufenden }\end{array}$ & Dateien mit einer Grösse über $350 \mathrm{~KB}$ \\
\hline $\begin{array}{l}\text { Gespräche, in denen mixed } \\
\text { code-Phänomene vorkom- } \\
\text { men }\end{array}$ & $\begin{array}{l}\text { Gespräche, in denen mixed } \\
\text { code-Phänomene vorkom- } \\
\text { men }\end{array}$ \\
\hline $\begin{array}{l}\text { Sämtliche Gespräche des Moderators } \\
\text { Rainer Maria Salzgeber mit den al- } \\
\text { lochthonen Experten an dreizehn der } \\
\text { neunzehn Spieltagen. }\end{array}$ & $\begin{array}{l}\text { Belegsammlung ,Mixed code in der } \\
\text { Schriftlichkeit" } \\
\text { Unsystematisch gesammelte Belege } \\
\text { unterschiedlicher Ausprägung aus } \\
\text { verschiedenen Quellen und Textsor- } \\
\text { ten. }\end{array}$ \\
\hline
\end{tabular}

Eine der grundlegenden Fragen zu Beginn der Korpuszusammenstellung war die Frage danach, ob sich das Vorkommen von sprachlichen Mischphänomenen zwischen Dialekt und Standardsprache in der Deutschschweiz mit einem Datenkorpus belegen lässt. Dabei sollte uns eine genügend grosse Datenmenge zur Verfügung stehen, die aus verschiedenen gesellschaftlichen Bereichen stammt. Wie bei jeder empirischen Untersuchung steht auch hier die

\footnotetext{
${ }^{8}$ Dialektelemente wurden kursiv gesetzt.
} 
Datensammlung im Spannungsfeld zwischen den Anforderungen an die Authentizität und Natürlichkeit der Daten auf der einen Seite und an die Wahrung von Persönlichkeitsschutz und Datenschutzbestimmungen auf der anderen. Auch ist sie abhängig vom Goodwill und Entgegenkommen der angefragten Institutionen, die in der Lage wären, dem Projekt Datenmaterial zur Verfügung zu stellen. Das bis zum jetzigen Zeitpunkt entstandene Korpus ermöglicht keinen Einblick in einen Querschnitt der sprachlichen Verwendung in der Deutschschweiz. Vielmehr sind hier einzelne Bereiche vertreten, die Belege unterschiedlicher Ausprägung liefern. Die einzelnen Teilkorpora ("Polizeinotruf"/"GFS-Kontaktgespräche"/"Euro 08-Studiogespräche"/"Sammlung schriftlicher Belege") ergänzen einander aber und ermöglichen es, verschiedene Facetten dieses bisher kaum beschriebenen Phänomens zu erfassen. Ihre Auswertung erfordert jedoch in jedem einzelnen Fall unterschiedliche methodische $\mathrm{Zu}$ gänge, was auch Auswirkungen auf die Vergleichbarkeit der Teilkorpora untereinander haben wird.

Als eine weitere Schwierigkeit erweist sich die theoretische Einordnung der untersuchten sprachlichen Erscheinungen. Aus den sehr unterschiedlichen, z. T. widersprüchlichen Theorien über code-switching, code-mixing, style-shifting etc. ${ }^{9}$ muss eine Auswahl getroffen und ein theoretisches Instrumentarium zusammengestellt werden, mit dem sich diese Phänomene beschreiben lassen. Der Abschluss der Korpuszusammenstellung wird demnach erst nach einer ersten Analyse und theoretischen Einordnung erfolgen können.

Die Prozessualität von Gesprächen und die Komplexität der für die Ausprägung der Sprachverwendung verantwortlichen Faktorenbündel machen es überdies schwierig, einzelne Grössen auszusondern und für statistische Berechnungen zu verwenden. Dementsprechend erscheint eine eingehende quantitative Auswertung der beschriebenen sprachlichen Erscheinungen als wenig sinnvoll. Auch erfordert die Suche nach Belegen aus der grossen Datenmenge der GFS-Kontaktgespräche eine Vorsortierung der Daten nach Grösse, denn die Gesprächslänge hat sich bereits bei der Untersuchung der Polizeinotrufgespräche als ein wichtiger Hinweis auf den Interaktionsgrad erwiesen, wobei das Auftreten der gesuchten Mischphänomene nur bei vorhandener Interaktion, also in längeren Gesprächen, zu erwarten ist. ${ }^{10}$ Dieses Vorsortieren wirkt sich jedoch unmittelbar auf die quantitativen Verhältnisse im Korpus aus und verfälscht sie. Um einige Aussagen über die Verteilung und Grössenordnung der untersuchten Phänomene formulieren zu können, wäre es jedoch denkbar, einen Teil der Daten unsortiert durchzuhören, um so exemplarisch einige quantitative Ergebnisse präsentieren zu können.

\section{$4 \quad$ Fazit}

Die hier vorgestellte Untersuchung von Mischphänomenen im Spannungsfeld zwischen Dialekt und Standardsprache in der Deutschschweiz basiert auf einem heterogenen Korpus. Seine Zusammenstellung war auf das Ziel hin ausgerichtet, eine möglichst breite Beleglage einiger seltener und bisher wenig beschriebener Erscheinungen zu erreichen. Das bisher entstandene Korpus ist insofern ausgewogen, als darin verschiedene Gesprächstypen und unterschiedliche

\footnotetext{
9 Je nach theoretischer Ausrichtung der verschiedenen Sprachkontaktforscher- und forscherinnen (als Hauptvertreter der verschiedenen Richtungen seien hier u. a. Peter Auer, Carol Myers-Scotton und Pieter Muysken genannt) werden die gleichen Phänomene als code-switching oder code-mixing beschrieben, oder aber wird die Existenz einer der beiden Erscheinungen ausser Acht gelassen. Auch die Diskussion um die sog. "mixed languages" erweist sich in diesem Zusammenhang als wenig fruchtbar, da es sich hier nicht um die Entstehung einer eigenständigen, konventionalisierten Varietät handelt, die von einer in sich geschlossenen gesellschaftlichen oder Volks-Gruppe verwendet wird (näheres dazu in: Matras, Yaron/Bakker, Peter (2003): The Mixed Language Debate. Theoretical and Empirical Advances. Berlin: de Gruyter. (= Trends in Linguistics 145).)

${ }^{10}$ Es handelt sich hierbei also um eine "Austarierung des Korpus nach internen Kriterien", die dem Zweck dient, "interessante linguistische Phänomene in ausreichendem Maße" zu dokumentieren (Lemnitzer/Zinsmeister 2006: 53).
} 
Sprecherkonstellationen in den einzelnen Teilkorpora vertreten sind. Die Belegsammlung aus der Schriftlichkeit dient als Ergänzung dazu. Ihr Vorteil gegenüber von durch Stichproben aus einem grösseren Textkorpus gewonnenen Einzelbelegen ist die Vielfalt der Beispiele und der vertretenen Textsorten. Es handelt sich dabei aber natürlich um eine unsystematisch gewonnene Sammlung, die keinerlei Aussagen über die quantitativen Verhältnisse erlaubt. Doch das Korpus erfasst auch insgesamt nur einen Ausschnitt der Realität und wird keine Aussagen über die wirkliche Verteilung und Grössenordnung der untersuchten sprachlichen Verwendungen erlauben. Insofern bleibt das schwer zu lösende Problem der Repräsentativität des Korpus auch in dieser Untersuchung bestehen. Andererseits gilt vielleicht in Bezug auf die von uns untersuchten Phänomene mehr denn je der Einwand, dass "die Repräsentativität einer Stichprobe im Verhältnis zur Grundgesamtheit eine Fiktion [ist], solange die Grundgesamtheit nicht exakt bestimmt werden kann" (Lemnitzer/Zinsmeister 2006: 53). Das uns zur Verfügung stehende Korpus wird es jedoch möglich machen, das Auftreten von Mischphänomenen zwischen Dialekt und Standardsprache zu belegen, erste Aussagen darüber zu formulieren, in welchen Kontexten diese vorkommen, welche Ausprägung sie haben und was ihre Verwendung auszeichnet und sie so in die wissenschaftliche Diskussion einzubringen.

\section{Literatur}

Auer, Peter (1986): "Kontextualisierung". Studium Linguistik 19: 22-47.

Auer, Peter (1999): "From codeswitching via language mixing to fused lects: Toward a dynamic typology of bilingual speech". International Journal of Bilingualism 3: 309-332.

Ammon, Ulrich (1995): Die deutsche Sprache in Deutschland, Österreich und der Schweiz. Das Problem der nationalen Varietäten. Berlin/New York: de Gruyter.

Ammon, Ulrich et al. (2004): Variantenwörterbuch des Deutschen. Die Standardsprache in Österreich, der Schweiz und Deutschland sowie in Liechtenstein, Luxemburg, Ostbelgien und Südtirol. Berlin/New York: de Gruyter.

Bürkli, Beatrice (1999): Sprachvariation in einem Grossbetrieb. Eine individuenzentrierte Analyse anhand sprachlicher Tagesläufe. Tübingen/Basel: Francke. (= Basler Studien zur deutschen Sprache und Literatur 73).

Christen, Helen (2000): "Standardsprachliche Varianten als stilistische Dialektvarianten?". In: Häcki Buhofer, Annelies (ed.): Vom Umgang mit sprachlicher Variation. Soziolinguistik, Dialektologie, Methoden und Wissenschaftsgeschichte. Tübingen/Basel, Francke: 245260.

Christen, Helen et al. (in Vorbereitung): Hochdeutsch in aller Munde. Eine empirische Untersuchung zur gesprochenen Standardsprache in der Deutschschweiz.

Dirim, Inci/Auer, Peter (2004): Türkisch sprechen nicht nur die Türken. Über die Unschärfebeziehung zwischen Sprache und Ethnie in Deutschland. Berlin/New York: de Gruyter.

Ferguson, Charles A. (1959): "Diglossia". Word 15: 325-340.

Gumperz, John J. (1982): Discourse strategies. Cambridge: Cambridge University Press.

Haas, Walter (2004): "Die Sprachsituation in der deutschen Schweiz und das Konzept der Diglossie". In: Helen Christen (ed.): Dialekt, Regiolekt und Standardsprache im sozialen und zeitlichen Raum. Wien, Praesens: 81-110.

Häcki Buhofer, Annelies/Häusermann, Jürg (1982): "Kontrast Mundart - Standardsprache". In: Burger, Harald/Häcki Buhofer, Annelies/Sialm, Ambros (eds.): Handbuch der Phraseologie. Berlin/New York, de Gruyter: 274-289.

Kloss, Heinz (1980): "Abstandsprachen und Ausbausprachen". In: Göschel, Joachim et al. (eds.): Dialekt und Dialektologie. Wiesbaden, Steiner: 301-322.

Kolde, Gottfried (1981): Sprachkontakte in gemischtsprachigen Städten. Vergleichende Untersuchungen über Voraussetzungen und Formen sprachlicher Interaktion verschiedensprachlicher Jugendlicher in den Schweizer Städten Biel/Bienne und Fribourg/Freiburg $i$. Ue. Wiesbaden: Steiner. (= ZDL-Beihefte NF 37). 
Labov, William (1972): Sociolinguistic Patterns. Philadelphia: University of Pennsylvania Press.

Lemnitzer, Lothar/Zinsmeister, Heike (2006): Korpuslinguistik. Eine Einführung. Tübingen: Narr.

Schuppenhauer, Claus/Werlen, Iwar (1982): "Stand und Tendenzen in der Domänenverteilung zwischen Dialekt und deutscher Standardsprache". In: Besch, Werner et al. (eds.): Dialektologie. Ein Handbuch zur deutschen und allgemeinen Dialektforschung. Bd. 2. Berlin, de Gruyter: 1411-1427.

Steger, Hugo et al. (1974): "Redekonstellation, Redekonstellationstyp, Textexemplar, Textsorte im Rahmen eines Sprachverhaltensmodells. Begründung einer Forschungshypothese". In: Moser, Hugo et al. (eds.): Gesprochene Sprache. IDS-Jahrbuch 1972. Düsseldorf, Pädagogischer Verlag Schwann: 39-97. (= Sprache der Gegenwart 26).

Werlen, Iwar (1988): "Swiss German Dialects and Swiss Standard High German. Linguistic variation in dialogues among (native) speakers of Swiss German dialects". In: Auer, Peter/Di Luzio, Aldo (eds.): Variation and Convergence. Berlin/New York, de Gruyter: 94-124. 